\title{
"Dimmi di che morte dobbiamo morire": dinamiche di persuasione strutturale nel post-terremoto del Centro Italia (2016-2017)
}

"Tell me what we're up against»: the dynamics of structural persuasion in the aftermath of an earthquake (Central Italy 2016-2017)

\section{Francesco Danesi}

\section{OpenEdition} Journals

Edizione digitale

URL: http://journals.openedition.org/aam/2582

DOI: $10.4000 /$ aam.2582

ISSN: 2038-3215

\section{Editore}

Dipartimento Culture e Società - Università di Palermo

\section{Notizia bibliografica digitale}

Francesco Danesi, «"Dimmi di che morte dobbiamo morire": dinamiche di persuasione strutturale nel post-terremoto del Centro Italia (2016-2017)», Archivio antropologico mediterraneo [Online], Anno XXIII, n. 22 (1) | 2020, online dal 20 juin 2020, consultato il 27 janvier 2021. URL: http://

journals.openedition.org/aam/2582 ; DOI: https://doi.org/10.4000/aam.2582

Questo documento è stato generato automaticamente il 27 janvier 2021.

\section{c) (†)}

Archivio antropologico mediterraneo è distribuita con Licenza Creative Commons Attribuzione - Non commerciale - Non opere derivate 4.0 Internazionale. 


\title{
"Dimmi di che morte dobbiamo morire": dinamiche di persuasione strutturale nel post-terremoto del Centro Italia (2016-2017)
}

\author{
"Tell me what we're up against»: the dynamics of structural persuasion in the \\ aftermath of an earthquake (Central Italy 2016-2017)
}

Francesco Danesi

1 Tra agosto 2016 e gennaio 2017, una serie di violente scosse di terremoto devastò il Centro Italia, colpendo numerose località delle regioni Marche, Umbria, Lazio e Abruzzo. Gli eventi sismici del 26 e del 30 ottobre, in particolare, sortirono effetti estremamente distruttivi. Circa un anno dopo cominciai a interessarmi del terribile disastro che il sisma aveva prodotto nei territori dell'Appennino Centrale. Pianificai dunque una ricerca etnografica, con l'obiettivo di indagare le complesse stratificazioni di una tragedia che aveva letteralmente sconvolto l'identità, le relazioni sociali e la quotidianità di numerose persone ${ }^{1}$. I miei spostamenti gravitavano attorno alla città di Macerata, presso la quale avevo trovato ospitalità da una mia collega; per circa tre mesi, a partire da settembre 2017, ebbi modo di visitare diversi paesi e borghi del "cratere" umbro-marchigiano; ascoltai le storie di numerosi "terremotati", i loro ricordi, la loro sofferenza, le loro speranze.

Ricordo che, durante l'impostazione del lavoro sul campo, mi fu dato un avvertimento: «Guarda che i marchigiani son tosti, eh!». Iniziai così, con una certa preoccupazione, a organizzare i miei sopralluoghi nelle regioni dell'Appennino Centrale, cercando di capire quale fosse il modo migliore per stabilire dei contatti. I mass media e le televisioni, infatti, avevano già consumato - dal punto di vista giornalistico - alcune delle principali località colpite, motivo per cui le popolazioni locali cominciavano a stancarsi di essere trattate come meri "fenomeni mediatici". Non sempre, in effetti, fu semplice stabilire un dialogo proficuo con i comitati o le associazioni a cui cominciai a rivolgermi. Spesso, inoltre, dovetti chiarire la natura della mia posizione, in quanto 
ricercatore-antropologo, nonché le domande che avevano spinto un "giovane bergamasco" ad esplorare le zone più recondite del cratere sismico. Volendo provare a penetrare e afferrare la complessità processuale del disastro (Hoffman, Oliver-Smith 2002), sin dall'inizio decisi di adottare una postura di ricerca "mobile": stabilii diversi contatti con alcune associazioni locali impegnate nella gestione dell'emergenza, cui poi seguì una vera e propria attività di ricerca itinerante. Attraverso un approccio etnografico di osservazione multi-situata ho potuto così raccogliere diverse forme di testimonianze, a partire dalle narrazioni dei terremotati sfollati fino alle rivendicazioni di attori sociali committed, cioè legati a comitati territoriali attivamente impegnati nella salvaguardia delle comunità colpite. Da tale approccio è derivato un buon numero di interviste in profondità - 17 quelle registrate e trascritte integralmente -, le quali sono state condotte con informatori selezionati per la loro predisposizione e apertura nei confronti della ricerca. I dialoghi hanno riguardato principalmente le loro storie personali, a partire dal ricordo delle scosse sismiche fino ai fatti più recenti legati al processo di ricostruzione; in particolar modo, alcune tematiche, legate alla disgregazione delle comunità, all'assenza di quotidianità e alla nostalgia del passato, sono risultate tra le più discusse e approfondite. Nondimeno, nelle conversazioni è stato affrontato anche l'intervento delle istituzioni, rispetto al quale ho cercato di approfondire le rappresentazioni locali, le strategie di adattamento e le conseguenze che il dispositivo d'emergenza, in seguito al terremoto, stava producendo. Pur non avendo proceduto a un'effettiva mappatura dei processi istituzionali, rispetto a questo tema ho cercato di ricomporre un quadro analitico di più ampio respiro attraverso la comparazione con altri contesti "post-terremoto" in Italia.

3 La visibilità della distruzione materiale prodotta dal disastro cela quasi sempre una serie di fatti socio-culturali che, rispetto allo spazio-tempo dell'evento specifico, si estende ben oltre la portata delle narrazioni egemoniche. L'analisi antropologica non solo permette di chiarire le condizioni di vulnerabilità storica e sociale su cui agisce un particolare agente distruttivo (Ligi 2009), ma può fornire anche un'utile chiave di lettura di realtà complesse, la cui trama di ragioni, rappresentazioni e significati presenta spesso un intreccio enigmatico. Valorizzare le narrazioni locali - narrazione intesa come dispositivo di costruzione del sé e del mondo (Kleinman, Kleinman 1991) significa attivare un vero e proprio confronto dialogico tra esperienza, incorporazione del mondo (Csordas 1990) e soggettivazione della storia; se possibile, significa comprendere il caos esperienziale che un disastro può determinare, sia a livello individuale sotto forma di crisi di presenza (de Martino 1977, 1995), sia a livello collettivo come rischio di disgregazione inesorabile della socialità e delle comunità locali (Hoffman, Oliver-Smith 2002; Ligi 2009). Tuttavia, l'indagine etnografica ha il dovere di spingersi oltre, fino a sondare le complesse relazioni tra implicazioni locali e interazioni globalizzanti della contemporaneità (Eriksen 2017; Friedman 1990; Giddens 1994; Kearney 1995), disvelando così le eventuali dinamiche persuasive che concorrono alla definizione di una contingenza sociale marginalizzante, le quali si rendono più evidenti proprio là dove si trova una situazione di vulnerabilità (Farmer 2004; Fassin 2013; Klein 2007; Saitta 2015). Nello specifico, le criticità identificate durante la ricerca etnografica sono state calate dagli interlocutori entro un discorso che problematizza, con toni più o meno estremi, la presunta strategia governativa di un abbandono intenzionale; tuttavia, $\mathrm{i}$ fatti contingenti del post-terremoto presentano una complessità che non può essere ridotta a una polarizzazione così netta $e$, anzi, configurano una serie di processi relazionali che, nel contesto di sospensione e 
ambiguità generato dal dispositivo d'emergenza, richiamano il concetto di "soglia di indecidibilità" (Agamben 2003). Attraverso la comparazione analitica con altri contesti post-sisma, si cercherà così di affrontare la situazione del Centro Italia mettendo in luce la contraddittorietà delle dinamiche persuasive che, nel prolungamento dello stato di emergenza, agiscono sullo spazio, sulla memoria e sui significati del disastro.

\section{La strategia dell'abbandono}

4 Pare che l'espressione "strategia dell'abbandono" sia apparsa per la prima volta nell'ottobre 2017, in un articolo del blog Lo Stato delle Cose, firmato da Leonardo Animali ${ }^{2}$. La teoria, secondo la quale lo Stato, nella fase dell'emergenza e della ricostruzione delle località colpite, sarebbe intervenuto volutamente a rilento, fu senza dubbio uno dei temi più discussi dai miei interlocutori. A Macerata incontrai Marco, un volontario di quarant'anni, affiliato all'associazione TerreInMoto Marche, con il quale approfondii alcune delle problematiche legate al processo di ricostruzione. Fu molto chiaro nell'illustrarmi la sua idea:

Quando magari sul blog di TerreInMoto, tu leggi "strategia dell'abbandono", queste cose qui... Ora ovviamente questa cosa noi la consideriamo non come che ci sia un... disegno, una mente dell'uomo grigio che sta lì a spostare le pedine, no? Come se fosse Risiko, però è evidente che lo spopolamento della montagna è un processo... che è già da tempo che è in atto, diciamo da dopo la fine della seconda guerra mondiale, naturalmente dopo gli anni Sessanta, dopo il boom. Però è anche vero che il terremoto in questo caso si trasforma in un acceleratore di questo processo, ok? (intervista a Marco, 17 ottobre 2017).

Come vedremo, le interpretazioni delle popolazioni locali sono diverse e variegate; in ogni caso, tale strategia sarebbe evidentemente legata - seguendo Marco - al lungo processo di spopolamento dei territori montani, che dalla fine del boom economico degli anni Sessanta starebbe consumando le zone dell'Appennino centrale ${ }^{3}$. Il terremoto e le conseguenze del disastro diventerebbero dunque degli acceleratori di tale processo, portando alla luce le problematiche che segnano queste zone dell'Italia da anni: la centralizzazione dei servizi lungo la costa - che permetterebbe una notevole riduzione dei costi amministrativi -, la mancanza di politiche pubbliche assennate e il disinteresse per le realtà a bassa densità demografica. Le rappresentazioni, l'immaginario e il vasto apparato di significati che le persone colpite dal sisma attribuiscono all'evento, nonché alle fasi immediatamente successive, possono in effetti gettar luce sul rapporto che intercorre tra le zone colpite e il "centro" politico (Saitta 2015). L'idea di "abbandono" rivela dunque un aspetto cruciale di questo rapporto: le popolazioni terremotate si trovano costrette in una situazione di passività, di attesa $\mathrm{e}$ di sconforto. L'atteggiamento degli apparati statali nella gestione della fase di ricostruzione e le relazioni che questi instaurano con le località distrutte chiarirebbero quindi la centralità o la perifericità delle aree afflitte, le quali possono - o non possono - essere in grado di negoziare e far valere i loro interessi (Ibidem).

6 Ad Amandola, un piccolo paese dell'entroterra marchigiano, in provincia di Fermo, trascorsi del tempo nella fattoria di Annalisa, una giovane allevatrice di 34 anni. La casa della sua famiglia era stata pesantemente danneggiata dalle scosse sismiche e arrivando dalla strada le crepe nei muri dell'abitazione erano ben visibili. La stalla, invece, era stata costruita secondo criteri anti-sismici innovativi e aveva resistito al terremoto, riuscendo così a proteggere gli animali e, soprattutto, permettendo alla famiglia di 
Annalisa, superate le prime fasi dell'emergenza, di riprendere le attività lavorative. In ogni caso, per due mesi e mezzo furono costretti a convivere all'interno di un camper, e solo nel mese di dicembre 2016, finalmente, la Regione Marche inviò la soluzione abitativa d'emergenza prevista per gli allevatori. Quando la vidi rimasi indubbiamente perplesso: si trattava di un container di dimensioni minuscole. All'interno la vivibilità era ridotta ai minimi termini: le pareti erano troppo sottili per sostenere i rigori dell'inverno, inoltre i materiali scelti rendevano il clima all'interno del modulo esageratamente umido, favorendo la comparsa di pozze assai poco salutari. Scoprii inoltre che inizialmente il container fu inviato vuoto e che i mobili, insieme agli elettrodomestici, sarebbero arrivati solo successivamente, in una data imprecisata. Allo stesso modo, sebbene il modulo fosse stato predisposto per gli allacci di luce e acqua, per evitare l'interruzione dell'elettricità sarebbe stato necessario potenziare il contatore fino a 6 kilowatt. La famiglia di Annalisa decise di ignorare le indicazioni delle istituzioni e, pur di abbandonare il camper, decise di arredare il container con $\mathrm{i}$ mobili che riuscirono a estrarre - illegalmente - dalla casa, inclusi i fornelli e una bombola di gas:

Ci siamo incaponiti noi [perché il container ancora non era pronto per essere abitato], abbiamo tirato fuori i nostri mobili dalle finestre, perché... tutto quello che vedi di questo colore qui [grigio] è dato dalla regione, tutto ciò che è di legno vero, il divano [ride], sono i nostri mobili che abbiamo fatto uscire dalle finestre, tirato fuori col trattore, eccetera. Perché? Questa il 16 dicembre era pronta, la ditta che doveva portare i mobili è scomparsa, non solo qui eh, è scomparsa nella zona di Arquata, dappertutto (intervista ad Annalisa, 2 novembre 2017).

7 Annalisa cercò di contattare gli uffici delle istituzioni preposte alla gestione dei moduli abitativi, inviando numerose e-mail e lettere, ma ogni tentativo si rivelò un buco nell'acqua. Oltretutto, i problemi non riguardavano unicamente il container fornito. Passeggiando nella fattoria notai che persino le aree di lavorazione della carne erano rimaste danneggiate. Annalisa mi spiegò che per continuare le attività lavorative avevano dovuto appoggiarsi al laboratorio del mattatoio presso il quale si servivano. La Regione Marche, in effetti, non aveva predisposto alcuna misura per garantire la ripresa degli allevamenti - nonostante la segnalazione di diversi enti (Ispettorato agrario, Guardia Forestale, Comuni) in merito alla necessità di moduli lavorativi ad uso macelleria - e successivamente fu addotta come giustificazione la mancanza di risorse economiche per sostenere una tale dotazione. Di Amandola e della fattoria ricordo molto bene tre cose: lo sguardo arrabbiato di Annalisa, l'espressione severamente malinconica del padre e i sorrisi serenamente disillusi della madre.

Silvio, un altro attivista della rete TerreInMoto Marche, aveva molto a cuore tutte queste problematiche; durante la nostra intervista si comportava come qualcuno che da mesi ripeteva ormai le stesse cose, e che tuttavia non voleva arrendersi. Mi spiegò così il suo punto di vista:

Cioè, non è che ci stanno i grandi vecchi che stanno chiusi in una stanza, e stanno lì: "Adesso io spopolo Ussita», no? Però è anche vero che, siccome i territori dell'Appennino sono abbandonati da anni, da anni gli vengono tolte risorse, gli vengono tolti i servizi... adesso c'è il terremoto, non si interviene, non ci stanno i centri di aggregazione, non ci sta... poi se fai uno-più-uno-più-uno-più-uno... non vuoi chiamarla strategia? Chiamala deriva verso, chiamala tendenza, chiamala convenienza verso l'abbandono. Chiamala come vuoi, però somiglia a una strategia, cioè di fatto c'è un'idea per cui quei territori mi costa troppo portarci i servizi, comunque c'è poca gente e quindi mi interessa poco, devo farci un certo tipo di interventi invasivi, quindi meno gente c'è, meglio è, tipo il gasdotto della SNAM, 
che deve passare proprio lì sotto. Sono tutte robe che comunque, ripeto, sommi tutte le varie componenti e alla fine quello è (intervista a Silvio, 31 ottobre 2017).

La molteplicità e la simultanea compresenza di diversi problemi, secondo Silvio, sarebbero la prova lampante di un difetto a livello sistemico o, più probabilmente, di una volontà precisa. In ogni caso, l'abbandono volontario e pianificato delle popolazioni agisce in maniera sinistra, in modo sottile, quasi implicito: analizzando a uno a uno gli aspetti critici dello stato di emergenza conseguente al terremoto, difficilmente sarebbe ipotizzabile un'interpretazione di tal tipo. È infatti la somma delle disattenzioni, dei ritardi e delle incongruenze che rende concreta la concezione di una strategia. Silvio prova anche a chiarire una possibile finalità di questo piano: la progettazione del gasdotto SNAM e gli interventi invasivi necessari per la sua attuazione, ad esempio, potrebbero in effetti motivare la scarsa volontà dello Stato nel garantire una rapida ricostruzione e messa in sicurezza dei luoghi terremotati ${ }^{4}$. L'urbanistica del disastro e della ricostruzione, nonché la burocrazia dell'emergenza, possono quindi rivelare gli eventuali interessi pubblici o privati in gioco, la cui tutela è regolata dai rapporti di forza dei diversi attori sociali coinvolti. Le relazioni sociali, politiche ed economiche che si instaurano nel disastro sono dunque esemplificative delle opportunità che il disastro stesso offre: l'abbandono - o il disimpegno, come ha già notato Irene Falconieri (2011) nello studio di un'alluvione in provincia di Messina del 2009 - non sarebbe altro che una modalità oscura dello Stato di dar forma ai suoi progetti, avvalendosi dell'eccezionalità di uno stato di emergenza che sospende la norma e afferma un tipo di governo delle popolazioni pressoché svincolato dallo stato di diritto precedente (Saitta 2015).

10 Alcuni dei terremotati di cui raccolsi le impressioni furono in ogni caso molto più cauti, se non addirittura scettici, rispetto all'idea di un'intenzionalità strategica. In tal senso, la retorica legata alla strategia dell'abbandono risulta ambivalente: da un lato diviene fondante delle rivendicazioni delle popolazioni locali, dall'altro può gettarle nello sconforto di un disegno prestabilito che non lascia via di scampo per il futuro. La complessità della situazione del post-terremoto, nel suo esser ridotta a "disegno", rischia di invalidare semplicisticamente le dinamiche della relazione tra le comunità $\mathrm{e}$ lo Stato. Tale relazione si gioca anche, in effetti, sul rapporto tra la politica locale delle località colpite e il governo centrale. Ne parlai con Emanuele, un lavoratore ultrasessantenne residente a Pieve Torina, un piccolo comune in provincia di Macerata. La sua casa era diventata inagibile in seguito alle scosse di ottobre, così, pur contravvenendo alle normative delle istituzioni, decise di installare di tasca propria una piccola casetta su ruote dove poter trovare rifugio insieme alla sua famiglia. Visitammo insieme le poche aree del paese che non erano state dichiarate "zona rossa" e non potei fare a meno di notare che l'atmosfera ricordava quella di un villaggio fantasma. La costruzione delle SAE procedeva a rilento, così molti abitanti avevano deciso di lasciare il paese. Aveva inoltre contribuito all'abbandono del luogo il pesante ritardo (più di un anno) con cui la regione - grazie ad un fondo stanziato dalla Protezione Civile - aveva autorizzato la costruzione di un'area commerciale provvisoria, destinata alla delocalizzazione di alcune attività commerciali o professionali. Mi spiegò così il suo punto di vista:

Ho pensato anche io a questa cosa qui, che dietro tutto ci fosse una strategia ben precisa di impoverire il territorio... Certo, mantenere le piccole comunità, paesi di mille, millecinquecento abitanti, sicuramente ha dei costi. A parte impoverirlo... togliere la presenza dell'uomo creerà dei problemi in futuro... la desertificazione. 
Però, ho pensato che davvero ci fosse la volontà di... anche perché i politici nostri ragionano tutti in termini di voto. Questo è il bacino di voti, qui saranno ventimila persone. Ventimila persone è mezza Civitanova [Marche], insomma, no? È un quartiere di Ancona (intervista a Emanuele, 30 ottobre 2017).

11 Egli, in pochissime parole, rende conto della situazione di subalternità che caratterizza le amministrazioni locali delle comunità: in un territorio che, dal punto di vista demografico, ha scarso peso politico, i politici locali intraprendono scelte orientate unicamente in base al voto e al consenso, cercando l'appoggio delle forze politiche dominanti a livello nazionale. Un altro intervistato, mentre discorrevamo di alcuni fatti della politica locale di Camerino, commentò così la situazione: «Non c'è più un senso delle istituzioni e il confronto sulle scelte. Le scelte vengono fatte per ragioni di quello che può essere il consenso per il singolo. Questo è uno dei tantissimi elementi che condizionano le scelte» (intervista a Bartolomeo, 16 ottobre 2017). Venendo meno il confronto con la popolazione, i politici locali agiscono alla ricerca del gradimento momentaneo - soprattutto sul piano mediatico -, legandosi alla retorica, alle direttive e alle visioni degli schieramenti politici più forti del governo nazionale; oppure, più semplicemente, optano per la cautela e l'attendismo, temendo di restare esclusi dalla già debole rete di attenzioni istituzionali. In un certo senso la politica locale finisce per amplificare le strategie di intervento e di ridefinizione del territorio prima, durante e dopo il disastro - anche a causa del rapporto di forza decisamente penalizzante per le comunità locali. Centralizzando il processo di ricostruzione, le possibilità di dialogo tra gli enti e le popolazioni locali si riducono drasticamente, con conseguenze che spesso sfidano la logica del buon senso e che assomigliano a tutt'altro che ad una strategia: basti pensare agli aumenti dei tassi di affitto nei centri urbani del cratere meno colpiti, all'avvilente trattamento che le comunità spostate negli hotel e nei centri di accoglienza lungo la costa hanno ricevuto alle soglie della stagione estiva del 2017, ai continui ritardi ed errori nella consegna delle strutture necessarie al ripristino delle attività economiche o alla disputa in merito all'abusività delle casette mobili adottate da molte persone come soluzione abitativa temporanea - preso atto delle scarse condizioni di vivibilità che offrono i container o le SAE (Soluzioni Abitative in Emergenza) fornite dalle regioni.

In sintesi: il disastro legittima l'instaurazione di una rete d'emergenza a cui si sottrae il normale ordinamento giuridico di diritto - possibilità, peraltro, prevista dal diritto stesso. La sovrapposizione delle istituzioni emergenziali alle amministrazioni locali corrisponde, di fatto, a una rottura degli equilibri - già precari - che regolano le relazioni tra le località del Centro Italia e il governo centrale, a favore di una centralizzazione decisionale che - almeno sulla carta - dovrebbe facilitare il processo di ricostruzione. L'eccezionalità del post-terremoto, tuttavia, garantisce anche una forma di azione svincolata dal diritto che rischia di accelerare o facilitare specifici progetti di ridefinizione geografica, sociale ed economica del territorio - come ben evidenziato da Pitzalis (2016) nel caso del terremoto emiliano del 2012. L'emergere di tali processi "trasformativi", che spesso prescindono dalla condizione emergenziale del disastro, dipende fondamentalmente dalla natura contraddittoria di un dispositivo d'emergenza che, a ben vedere, deve conciliare la salvezza della popolazione con la complessa rete di fini e motivazioni politiche ed economiche perseguite da particolari attori sociali, le cui interazioni, in ogni caso, non possono essere ridotte a un'intenzionalità monadica. 


\section{Soglie di indecidibilità}

13 Prima di procedere, vorrei soffermarmi brevemente sullo stato di sospensione della norma che caratterizza il post-terremoto, determinato dalla logica ambigua del dispositivo d'emergenza. Nel caso del Centro Italia il disastro ha determinato una modificazione radicale nella gestione della res publica, tanto sul piano dell'amministrazione politica locale quanto su quello della pianificazione da parte del governo nazionale. Lo stato d'emergenza è certamente assimilabile ad uno "stato di eccezione" (Agamben 2003) entro il quale le istituzioni operano e perseguono i loro fini liberamente, superando l'ordinamento normale del diritto. Tale descrizione, tuttavia, merita di essere problematizzata: è necessario, in via preliminare, comprendere l'ambiguità del dispositivo d'emergenza, osservandone e studiandone - per quanto possibile - l'esploso ${ }^{5}$. Si vuole qui evitare quelle semplificazioni o distorsioni teoriche che, come ha fatto notare Fabio Dei (Dei, Di Pasquale 2017), a partire dalla rigida applicazione del cosiddetto canone o paradigma epistemologico dell'antropologia critica contemporanea, rischiano di invalidare la bontà dell'etnografia e della riflessione antropologica ${ }^{6}$. Nello specifico, pur rispettando l'accezione foucaultiana del termine, vorrei evitare di ridurre il concetto di dispositivo a una sorta di congegno che opera secondo una procedura di input-output, finalizzata alla disciplina, al controllo e alla classificazione ${ }^{7}$; allo stesso modo, ritengo necessario problematizzare la nozione agambeniana di stato di eccezione calandola entro le dinamiche osservate a livello locale, così da verificarne l'effettivo potenziale interpretativo. Credo, cioè, che sia possibile adottare una prospettiva epistemologica meno plastica e più disponibile ad accogliere le contraddizioni insiste in uno specifico contesto sociale.

Nel post-terremoto del Centro Italia, la gestione governativa della ricostruzione ha sin da subito mostrato evidenti carenze, stimolando la nascita di una moltitudine di associazioni, comitati e movimenti locali impegnati - chi attraverso la protesta, chi attraverso il dialogo - nella rivendicazione di attenzioni e risorse. Oltre all'intervento della Protezione Civile, infatti, mirato alla messa in sicurezza della popolazione colpita, nelle fasi immediatamente successive alle scosse, sembra lecito affermare che non si è mai costituita una vera e propria rete di intermediazione tra le località colpite, le regioni e le istituzioni governative preposte alla gestione dell'emergenza - aspetto che quasi tutti i miei interlocutori hanno lamentato. La centralizzazione decisionale, che ha certamente estromesso dal dialogo sul disastro le popolazioni terremotate, per la verità ha assunto sin da subito tratti fantasmatici, determinando una situazione limbica in cui l'assegnazione delle responsabilità è rimasta in costante mutamento e spesso vincolata a dimensioni extra-emergenziali: mi riferisco qui alla sovrapposizione di interessi politici ed economici, pubblici e privati, esterni al disastro, che, lungi dall'essere il frutto di una pianificazione diabolica, è però resa possibile da questa condizione di indecidibilità generale. Dunque, non stupisce affatto che in alcune località la salvaguardia della popolazione sia il risultato di un'autogestione locale, che talvolta si colloca "al di fuori" della legge, o che le norme burocratiche per la ricostruzione, applicate nei territori dell'Appennino Centrale, siano totalmente incompatibili rispetto alle peculiarità delle singole situazioni ${ }^{8}$. Il cosiddetto dispositivo d'emergenza assomiglia più ad una chimera, una forma di ibridazione confusa, reale e spettrale, che finisce per concretizzare una serie di contraddizioni non semplicemente imputabili allo Stato - il quale, attraverso le sue diverse emanazioni istituzionali, come abbiamo detto, 
è per lo più assente: a farla da padrone, dunque, sono quelle reti di attori sociali, strutture e narrazioni, che controllano e riescono a catalizzare l'afflusso di risorse in una località piuttosto che in un'altra.

Più che sullo stato di eccezione, allora, credo sia necessario ragionare sull'idea di "soglia di indecidibilità" (Agamben 2003), la quale evoca, per l'appunto, una dimensione di indeterminatezza in cui la responsabilità, il diritto e la necessità sono costantemente catturati in una duplice relazione di assenza e presenza, a seconda dei contesti e degli attori sociali coinvolti nella rete relazionale dell'emergenza. Contrariamente a una visione che si fonda sull'intenzionalità di un "controllore" che opera senza vincoli, lo stare-sulla-soglia implica infatti la sfocatura di ogni vincolo, la dissoluzione di qualsivoglia status e la costante oscillazione tra il dentro e il fuori. Questo vale sia per i terremotati, la cui condizione di marginalità è radicata nell'indefinitezza temporale dello stato di emergenza, sia per le emanazioni del governo, la cui effettiva presenza ed efficacia non è mai accertabile in maniera definitiva, sia per quei soggetti istituzionali e privati che nel post-terremoto hanno la possibilità di sperimentare forme inedite di intervento nelle comunità e sul territorio. Resta difficile spiegare la ferrea volontà di quanti hanno deciso di restare nei luoghi distrutti, impegnandosi affinché la ricostruzione possa realmente avvenire o attendendo che si presenti un interlocutore credibile. Forse, questo stesso "stare a metà" stimola un bisogno simbolico che è legato alla necessità di possedere o riappropriarsi di un'identità riconoscibile. Non a caso $i$ movimenti nati a livello locale hanno spesso insistito su quest'aspetto, attraverso una continua riaffermazione delle specificità geografiche e culturali locali, e il rifiuto delle generalizzazioni scientifiche, mediatiche e istituzionali. Si tratta di forme di localismi identitari che sollevano, fondamentalmente, un interrogativo": siamo dentro o fuori? Nelle parole di uno dei miei informatori, Daniele, il quale da mesi era stato dislocato in un hotel sulla costa adriatica: «[...] Dimmi di che morte dobbiamo morire e almeno fatecelo sapere, mentre nessuno parla chiaro» (intervista a Daniele, 1 novembre 2017).

\section{La persuasione strutturale}

Il noto antropologo statunitense Paul Farmer introdusse nei primi anni Duemila il concetto di "violenza strutturale", utile a spiegare le pratiche violente esercitate sistematicamente da un particolare ordine sociale - violenza esercitata attraverso l'elisione della memoria storica e altre forme di desocializzazione, i cui risultati sono allo stesso tempo moralmente ingiustificabili e apparentemente privi di responsabilità chiaramente attribuibili (Farmer 2004). Egli invitava la comunità delle scienze sociali ad aprirsi alla "materialità del sociale", nei termini in cui qualsiasi progetto sociale richiede il proprio materiale di costruzione: è in tale processo che si manifesta storicamente la violenza strutturale, negli esiti avversi delle dinamiche costruttive, caratterizzati da marginalità, disagio, sofferenza, malattia e morte. Storicizzare la materialità del sociale, secondo le indicazioni di Farmer, significa rendere visibili le reti di potere nelle quali resta imbrigliata la miseria dei deboli; significa mostrare le modalità con le quali l'ineguaglianza viene strutturata e legittimata nel tempo; significa decostruire le narrazioni egemoniche della realtà e provare a ripristinare le connessioni tra fatti e luoghi apparentemente distanti tra loro nello spazio e nel tempo.

Nel caso di un disastro si manifestano le complesse relazioni che intercorrono tra quelle che il sociologo Giddens (1994) chiama "implicazioni locali" - o circostanze di 
compresenza - e "interazioni a distanza" - ossia le connessioni di presenza e assenza. La modernità capitalistica, sostiene Giddens, ha favorito l'isolamento della sfera economica da quella politica, creando un rapporto di dipendenza che, in ultima analisi, assecondando i paradigmi di mercato globali, ha dato luogo a metamorfosi geografiche e demografiche senza precedenti. Tale dinamica, in linea generale, risponde al quadro interpretativo della violenza strutturale. L'alterazione del rapporto dialettico tra locale e globale, tuttavia, non è semplicemente riducibile a un dualismo relazionale in cui dominano incontrastate le forze della globalità. Eriksen (2017) ha giustamente sottolineato l'importanza di studiare e comprendere le modalità con cui l'interconnessione politica ed economica del mondo contemporaneo si manifesta su scala locale, suscitando trasformazioni e conflitti di cui non sempre è possibile generalizzare le logiche. Rispetto al caso etnografico in questione intendo dunque discostarmi dalla terminologia adottata da Farmer, per una semplice ragione: nel contesto di un disastro non credo sia possibile riferirsi all'azione delle istituzioni e alle logiche che ne guidano l'intervento con termini presi a prestito dall'ambito semantico della violenza; il rischio concreto è quello di ipostatizzare entità quali lo Stato o il potere, rendendole astrazioni monolitiche connotate negativamente. Preferisco adottare l'idea di "persuasione strutturale": dal latino persuadeo, "convincere". La persuasione, infatti, chiarisce la natura dialettica e stratificata del complesso di rapporti tra mondi locali e contemporaneità globale - non sempre riconducibile a strutture egemoniche del potere - e racchiude in sé la dimensione della temporalità, poiché qualsiasi opera di persuasione richiede un tempo di negoziazione. Cercherò, in quest'ultima sezione del saggio, di rintracciare e descrivere le qualità fondamentali di questa idea, ricostruendone il codice genetico e genealogico, sulla base dei dati raccolti sul campo, nonché attraverso altri casi di disastro occorsi in Italia.

Il primo aspetto riguarda la composizione elementare del paradigma che orienta le azioni, le narrazioni e i significati di un disastro. In tal senso, di particolare interesse saranno le ideologie politico-economiche dominanti, la definizione delle istituzioni preposte alla gestione di un'emergenza, la quantificazione delle risorse disponibili, le modalità di intervento, le modalità di redistribuzione delle risorse e le modalità di partecipazione/estromissione, a loro volta definite da specifiche tecniche burocratiche, dialogiche e rappresentative. Il geografo tedesco Robert Geipel (1991) si occupò, ad esempio, del terremoto del Friuli verificatosi nel 1976, rilevando alcune criticità che nella memoria culturale del disastro sono state per lo più minimizzate: nella regione friulana colpita dal sisma, lo Stato italiano, infatti, dispose programmaticamente gli interventi ricostruttivi secondo una logica di espansione industriale senza precedenti. Gli ingenti investimenti nel settore edilizio e immobiliare, inoltre, dilatarono a dismisura lo spazio sociale di cui erano solite fruire le popolazioni dell'area colpita: spazio che, a causa dei fenomeni di emigrazione dalle zone terremotate, nelle periferie rimase completamente inutilizzato, e che per tale ragione provocò una complessiva svalutazione delle case, del lavoro e delle attività locali. A dispetto di tutto questo, la maggior parte della popolazione locale, soprattutto quella che partecipò direttamente alla ricostruzione, investendoci i propri risparmi personali, sembra ancora oggi essere soddisfatta dei risultati raggiunti; il miraggio della modernizzazione e dell'industrializzazione riuscì, insomma, a oscurare l'aggravamento delle condizioni sociali delle località colpite.

19 La seconda qualità che definisce la persuasione strutturale riguarda la capacità che certi discorsi, risultanti da una particolare composizione elementare, hanno di 
diffondersi in maniera epidemica, fino a diventare egemonici. Diffusione dunque di interpretazioni, rappresentazioni, sistemi simbolici inerenti al disastro, i quali non sono semplicemente calati dall'alto, da parte di uno Stato monolitico, ma sono sempre il risultato di un paradigma che è costantemente oggetto di negoziazione. Il caso del terremoto Irpino del 1980 - il cui processo di ricostruzione, per altro, non è ancora terminato - è particolarmente illuminante da questo punto di vista: il campo del discorso istituzionale sul terremoto, in questo caso, produsse e diffuse nel tempo una "verità" utile a mettere in luce l'arretratezza del Mezzogiorno d'Italia (D'Ascenzio 2015); l'evento, plasmando il senso comune della popolazione, giustificò, di fatto, un intervento delle istituzioni contro la miseria e la disoccupazione. Va situata in questo contesto l'azione della classe dirigente nazionale e delle lobby locali, che, attraverso una fitta rete clientelare (Caruso 2013), formata da imprenditori, politici e funzionari statali, assunse i tratti di un'economia della catastrofe, cui non mancò l'influenza di poteri trasversali di origine mafiosa. L'intervento dello Stato, attraverso la rete d'emergenza, si trasformò in una triangolazione viziosa tra politica locale, imprenditoria e malaffare (Ventura 2015), in cui gli obiettivi e gli interessi delle élite legate alla ricostruzione vennero ridefiniti e ricalibrati di volta in volta, secondo particolari strategie e tattiche relazionali (D'Ascenzio 2015), che di fatto hanno estromesso le frange più deboli della popolazione terremotata.

Il terzo ed ultimo elemento che costituisce la persuasione strutturale riguarda la capacità che le componenti di un particolare ordine sociale hanno di selezionare, nel dispiegarsi della storia, determinati discorsi, attraverso l'oscuramento o la sedazione di altre forme di rappresentazione e narrazione. I miei interlocutori hanno spesso rievocato il sisma umbro-marchigiano del 1997, generalmente ricordato come esempio di intervento positivo dello Stato. Alcuni di essi, tuttavia, mi fecero notare che anche in quel caso, purtroppo, si erano manifestate diverse contraddizioni. Marco, ad esempio, commentò così la ricostruzione di alcuni centri abitati:

Non so se tu hai visto nelle frazioni di Serravalle, Dignano, vicino all'altopiano di Colfiorito... Sono frazioni che fortunatamente, questa volta, non sono state colpite pesantemente. E tu lì arrivi e vedi la ricostruzione del '97. Ma tu vedi nuclei abitativi, di dieci, dodici case, frazioni, borghetti, contrade, completamente ristrutturati, perfettamente ristrutturati... vuoti (intervista a Marco, 17 ottobre 2017).

21 Lo studio etnografico di Enrico Marcorè, condotto presso Nocera Umbra, una delle località colpite, ha giustamente evidenziato alcune criticità legate alle politiche della ricostruzione, le quali, col trascorrere degli anni, non hanno fatto altro che accelerare il processo di spopolamento cui abbiamo già accennato in precedenza, risultando infine nei "borghi senza vita" (Marcorè 2015) a cui si riferisce Marco nel breve estratto. Marcorè, nella sua analisi di Nocera Umbra, a dodici anni dal lavoro di campo, scrive: «Riteniamo pertanto di poter affermare che, secondo un'analisi diacronica (seppur su breve durata), lo spopolamento del centro è stato rinvigorito pesantemente dal terremoto e definitivamente sancito dalle politiche di ricostruzione» (ibidem: 203). Eppure, attraverso la storia sono state selezionate le narrative mediatiche, istituzionali, politiche ed economiche, utili a plasmare una storia precisa di tale ricostruzione, eletta a modello positivo e generalmente rievocata dai terremotati odierni come strada da ripercorrere. I risultati di tali narrazioni, d'altronde, emergono chiaramente - giusto per fare un esempio - dalle parole di Daniele: «Noi avevamo chiesto, appunto, il sistema del '97, che aveva funzionato benissimo» (intervista a Daniele, 1 novembre 2017). 
La strategia dell'abbandono, riletta alla luce di quanto detto finora, rappresenta l'interpretazione di una serie complessa di fatti storici, culturali e socio-economici, in larga parte determinati dal rapporto tra politiche locali e politiche nazionali; si tratta di un'interpretazione colta nell'eccezionalità del momento critico del post-terremoto, in cui i singoli individui cercano di conferire ordine, motivazioni e significati agli eventi che li coinvolgono ${ }^{10}$. L'abbandono sarebbe cioè il frutto delle particolari modalità di gestione del dispositivo di emergenza da parte dello Stato, il cui atteggiamento, come abbiamo notato, è certamente ambiguo: centralizzando il potere decisionale, dilazionando nel tempo le scelte di ricostruzione, e riallocando la popolazione colpita altrove, si creano infatti, per alcune élite tecnico-burocratiche, le condizioni favorevoli per azioni speculative in grado di ridisegnare il territorio secondo quella che Klein (2007) definisce come "economia del disastro» - situazione in cui le possibilità di partecipazione, organizzazione e mobilitazione delle popolazioni locali sono ridotte ai minimi termini (Pirone, Rebeggiani 2015). Tale rappresentazione concretizza parzialmente, sul piano retorico, alcune delle criticità esperite dai terremotati, le quali sono riconducibili, in ultima analisi, alle dinamiche della persuasione strutturale. A tal proposito riprendo le parole di Silvio:

Le scuole: tutte le scuole che sono state ricostruite, sono state ricostruite con soldi privati. Total-Erg, Salini-Impregilo, tutti. Che è una roba terrificante. Uno, perché ci stanno a comprare e neanche ce ne rendiamo conto. Due, perché salta qualsiasi tipo di programmazione [...] Cioè, Amandola, i soldi per l'ospedale, li hanno dati una compagnia petrolifera russa che si chiama... oddio non mi ricordo il nome, comunque lo trovi. È la stessa compagnia petrolifera che è proprietaria del $60 \%$ di SNAM, che è quella che farà il gasdotto che passa lì sotto ${ }^{11}$. Il TAP, che poi qui cambia nome, diventa gasdotto SNAM, però è sempre la stessa. Anche lì, non è che voglio essere complottista, però due più due può far quattro virgola uno, ma non può fare otto (intervista a Silvio, 31 ottobre 2017).

Il nodo cruciale della narrazione istituzionale del post-terremoto, secondo Silvio, consiste nell'oscuramento delle forze che interagiscono con il processo di ricostruzione. La confusione e la mancata assegnazione di responsabilità certe tra pubblico e privato, in effetti hanno legittimato l'intervento autonomo di imprese e aziende multinazionali, spesso attivato dalle più o meno spiccate capacità relazionali degli amministratori pubblici. Più che di "complottismo", qui si tratta di richiamare l'attenzione, cogliendo gli spunti di Silvio, sulle inevitabili distorsioni che la configurazione chimerica del dispositivo d'emergenza, frutto delle sovrapposizioni e delle azioni che le soglie di indecidibilità rendono possibili, produce.

Lo storico Giacomo Parrinello (2015) ha brillantemente mostrato, analizzando il terremoto di Messina del 1908 e quello del Belice del 1968, come nell'estensione temporale di un disastro si creino le condizioni "strutturali" utili a modificare la geografia sociale e urbana di un territorio, secondo logiche che non sempre rispondono alla scala locale del fenomeno e che proprio per questo meritano di essere indagate storicamente. Non diversamente, in questo articolo ho cercato di problematizzare la spazialità e la temporalità del disastro alla luce delle complesse stratificazioni che, di fatto, concorrono a plasmare - o annullare - morfologicamente e semanticamente il fenomeno indagato, focalizzandomi in particolar modo sulle rappresentazioni emiche e sulle pratiche discorsive emerse in un contesto in cui l'emergenza sembra prolungarsi in maniera indefinita. Resta da capire, a tal proposito, come la composizione del paradigma di questo post-terremoto, caratterizzata dall'assenza e dall'interdetto, muterà nel tempo, considerate anche le peculiarità dell'azione fantasmatica delle 
istituzioni, i campi discorsivi entro cui precipitano le narrazioni sul disastro e le idiosincrasie che la condizione di soglia ha determinato fino ad oggi; ci si chiede, infine, quali fenomeni di diffusione e di selezione agiranno sulla storia del disastro e come verrà persuasa la memoria culturale dell'evento. Il rischio concreto è che, ancora una volta, l'"utile" di un particolare ordine sociale possa venire privilegiato, a discapito della sofferenza, della marginalità e del silenzio che l'incapacità cronica di affrontare le emergenze in Italia, forse imputabile alla mancanza di un discorso culturale "del" disastro, continua a produrre.

\section{Conclusioni}

Nel contesto del post-terremoto del Centro Italia è lecito affermare che le popolazioni colpite continuano a versare in condizioni di estrema vulnerabilità, la quale è determinata dalle peculiarità di un dispositivo d'emergenza ibrido che concretizza molteplici contraddizioni di natura politica ed economica; l'ambiguità che ne caratterizza gli effetti è dovuta, in parte, ai particolari processi di ridefinizione geografica e sociale del territorio che, pur prescindendo dalle contingenze e dalle urgenze del disastro, vengono attivati o accelerati da specifiche reti di attori sociali interni ed esterni al disastro. La "strategia dell'abbandono", dunque, intesa come campo del discorso che ha regolato le rappresentazioni locali, ha orientato la riflessione e le narrazioni della popolazione terremotata intorno a una spiegazione che, attraverso l'identificazione di un'intenzionalità chiara, intercetta alcune delle idiosincrasie insite nella gestione dell'emergenza. La necessità di attribuire un significato agli eventi del disastro, e di riconoscere delle responsabilità certe, è giustificata dalla soglia di indecidibilità che caratterizza lo stato d'emergenza, al cui interno i discorsi e le azioni istituzionali si fanno spettrali, secondo dinamiche di assenza e presenza che precipitano qualsiasi decisione nell'indeterminato - stimolando così le iniziative di movimenti, comitati e associazioni locali che, fondamentalmente, rivendicano l'attribuzione di uno status certo.

È possibile intravedere, nel caso illustrato, le dinamiche di un'azione che ho definito come "persuasione strutturale", risultante dal complesso di relazioni tra località e globalità che, nel dispiegarsi della storia, sono in grado di plasmare, ridefinire e risignificare il significato e la memoria culturale degli eventi. La ricostruzione della genetica di quest'idea, attraverso una sommaria genealogia dei fenomeni che la interessano, ci ha permesso di identificare tre qualità fondamentali: la composizione elementare del paradigma da cui, di volta in volta, scaturisce; la capacità di diffondere, rendendole dominanti, specifiche narrazioni degli eventi, che infine costituiscono campi egemonici del discorso; la possibilità di selezionare, attraverso l'esclusione e l'oscuramento, le rappresentazioni e le narrative che, col trascorrere del tempo, hanno un effetto scultorio sulla memoria e sulla storia. Per quanto riguarda il Centro Italia, in ogni caso, ci si domanda quali conseguenze avrà, tanto sul piano socio-culturale includendo il tema della memoria culturale -, quanto su quello più tangibile legato alla geografia del sisma, la presenza-assenza fantasmatica delle emanazioni statali a lungo termine. Infine, ritengo sia fondamentale riflettere sul ruolo che l'approccio antropologico può giocare, nei termini di un'applicazione integrata del suo metodo e del suo sapere, all'interno degli stessi processi istituzionali, politici ed economici che sono stati presi in esame in questo saggio; a tal proposito, non credo sia sconveniente 
auspicare una presa di coscienza, anche in ambito accademico, delle possibilità di un'antropologia votata alla mediazione, all'impegno e al pragmatismo - secondo una forma di partecipazione basata sulla professionalità, non sulla militanza o sull'attivismo - che potrebbe stimolare, supportare e favorire il dialogo tra rappresentanze locali, civiche e amministrative, e le istituzioni preposte alla gestione di una crisi emergenziale.

\section{BIBLIOGRAFIA}

Adornato F., Cegna A., 2013 Le Marche nella mezzadria. Un grande futuro dietro le spalle, Quodlibet, Macerata.

Agamben G., 2003 Stato d'eccezione, Bollati Boringhieri, Torino.

Agamben G., 2006 Che cos'è un dispositivo?, Nottetempo, Roma.

Bock J., 2017 «The Second Earthquake: How the Italian State Generated Hope and Uncertainty in Post-Disaster L'Aquila», in Journal of the Royal Anthropological Institute, Vol. 23 (1): 61-80.

Borghi E., 2017 Piccole Italie: le aree interne e la questione territoriale, Donzelli, Roma.

Bourdieu P., 2003 Per una teoria della pratica. Con Tre studi di etnologia cabila, Raffaello Cortina Editore, Milano (ed. or. 1972 Esquisse d'une théorie de la pratique précédé de Trois études d'ethnologie kabyle, Editions du Seuil, Paris).

Caruso T., 2013 «Trust, Clientelism and State Intervention in Disaster Relief Policy: The Case of Southern Italy», in Human Affairs, Vol. 23: 230-245.

Csordas T. J., 1990 «Embodiment as a paradigm for anthropology», in Ethos, vol. 18 (1): 5-47.

D’Ascenzio A., 2015 «Microcosmo imprenditoriale irpino: tra polvere e macerie», in Saitta (a cura di) (2015): 81-92.

Dei F., Di Pasquale C. (a cura di), 2017 Stato, violenza, libertà. La «critica del potere» e l'antropologia contemporanea, Donzelli, Roma.

de Martino E., 1995 Storia e metastoria. I fondamenti di una teoria del sacro, Argo, Lecce.

de Martino E., 1977 La fine del mondo. Contributo all'analisi delle apocalissi culturali, (a cura di Clara Gallini), Einaudi, Torino.

Dines N., 2016 «Ethnographic Reflections on 'Oppositional Heritage Discourse' in Two PostEarthquake Italian Cities», in International Journal of Heritage Studies, vol. 22 (2): 102-116.

Eriksen T. H., 2017 Fuori controllo. Un'antropologia del cambiamento accelerato, Einaudi, Torino (ed. or. 2016 Overheating. An Anthropology of Accelerated Change, Pluto Press, London).

Falconieri I., 2011 «Politiche pubbliche. Il caso etnografico di un comune della Sicilia Nordorientale», in Quaderno di comunicazione, vol. 12: 103-112.

Farmer P., 2004 «An anthropology of structural violence», in Current Anthropology, vol. 45, n. 3: 305-325. 
Fassin D., 2013 La forza dell'ordine. Antropologia della polizia nelle periferie urbane, La Linea, Bologna (ed. or. 2011, La force de l'ordre. Une anthropologie de la police des quartiers, Édition du Seuil, Paris).

Foucault M., 1972 L'ordine del discorso, Einaudi, Torino (ed. or. 1971, L'ordre du discours, Éditions Gallimard, Paris).

Friedman J., 1990 «Being in the World: Globalization and Localization», in Theory, Culture \& Society, vol. 7: 311-328.

Geipel R., 1991 Long-term Consequences of Disasters. The Reconstruction of Friuli, Italy, in Its International Context, 1976-1988, Springer-Verlag, New York.

Giddens A., 1994 Le conseguenze della modernità, Il Mulino, Bologna (ed. or. 1990, The Consequences of Modernity, Polity Press, Cambridge).

Hoffman S. M., Oliver-Smith A. (eds), 2002 Catastrophe \& Culture. The Anthropology of Disaster, SAR Press, Santa Fe.

Kearney M., 1995 «The Local and the Global: The Anthropology of Globalization and Transnationalism», in Annual Review of Anthropology, vol. 24: 547-565.

Klein N., 2007 Shock economy. L'ascesa dell'economia dei disastri, Rizzoli, Milano.

Kleinman A., Kleinman J., 1991 La sofferenza e la sua trasformazione professionale. Verso un'etnografia dell'esperienza interpersonale, in Quaranta (a cura di) (2006): 199-234.

Ligi G., 2009 Antropologia dei disastri, Laterza, Roma-Bari.

Marcorè E., 2015 «Nocera Umbra: diacronica di un disastro annunciato», in Saitta (a cura di) (2015): 197-208.

Parrinello G., 2015 Fault Lines. Earthquakes and Urbanism in Modern Italy, Berghahn Books, New York.

Piermattei S., 2007 Antropologia ambientale e paesaggio agrario, Morlacchi, Perugia.

Pirone F., Rebeggiani E., 2015 «Saperi e poteri nella gestione pubblica dell'emergenza: la presa in carico degli sfollati del terremoto all'Aquila», in Saitta (a cura di) (2015): 209-220.

Pitzalis S., 2016 Politiche del disastro. Poteri e contropoteri nel terremoto emiliano, Ombre Corte, Verona.

Saitta P. (a cura di), 2015 Fukushima, Concordia e altre macerie. Vita quotidiana, resistenza e gestione del disastro, Editpress, Firenze.

Ventura S., 2015 «Il terremoto in Irpinia del 1980: memorie individuali e collettive del sisma», in Saitta (a cura di) (2015): 187-196.

\section{NOTE}

1. Per le finalità di questo articolo alcuni nomi di persone, luoghi e associazioni legate alla ricerca sono stati cambiati, così da rispettare la delicatezza di alcune tematiche qui affrontate.

2. La prima parte recita: «La strategia dell'abbandono abita da sempre sull'Appennino. [...] Nel tempo, durante le fasi di quiete, la strategia dell'abbandono si alimenta di cattiva edilizia, saccheggio del paesaggio e delle risorse naturali, mancata prevenzione geomorfologica, e di patrimoni immobiliari lasciati all'incuria da eredi, che neanche si ricordano di essere proprietari di una casa della bisnonna (Leonardo Animali, 11 ottobre 2017, Così il terremoto ha ridato linfa alla strategia dell'abbandono, www.lostatodellecose.com, ultima consultazione: dicembre 2017)». 
3. Lo spopolamento delle aree interne degli Appennini è un fenomeno largamente studiato e documentato. Il declino demografico è iniziato nell'Ottocento andando di pari passo, sebbene a ritmi molto diversi tra il settentrione e il meridione, con la crescente industrializzazione del paese e la conseguente modificazione del paesaggio agrario (Piermattei 2007; Borghi 2017). Nel secondo Dopoguerra il processo subisce poi un'accelerazione legata a ragioni di riorganizzazione e centralizzazione urbana ed economica, le quali, nel caso specifico delle Marche, hanno ad esempio portato alla quasi totale sparizione del sistema mezzadrile (Adornato, Cegna 2013).

4. A proposito del gasdotto SNAM - o TAP -, Marco mi disse: «La TAP - no? - è questo gasdotto che parte dall'Azerbaijan e finisce in Austria, a noi non ci daranno nemmeno un metro cubo di gas, però passerà tutto interno ai Sibillini. Ora, a conti fatti, la ricostruzione, è stato detto, costa ventitré miliardi e mezzo di euro, per ricostruire tutto. [...] La TAP, se non ricordo male, costa intorno ai dodici miliardi di euro. [...] Perché, quando uno si sente rispondere "non ci sono i soldi", deve avere tutte le informazioni possibili per capire che... non è che non ci sono i soldi in generale. Si decide che quei soldi che ci stanno vengono orientati per altri scopi, perché...» (intervista a Marco, 17 ottobre 2017).

5. Con "esploso" mi riferisco metaforicamente a quel particolare disegno tecnico che raffigura separatamente le varie parti di una macchina o di un dispositivo, così da renderne più chiaro il funzionamento.

6. Dei, in particolare, si riferisce alla rigida applicazione della cosiddetta theory, composta da un canone disparato di autori come Marx, Foucault, Deleuze, Bourdieu, Agamben, Benjamin, Latour - per citarne alcuni -, la quale sarebbe funzionale a una critica radicale dello Stato e del potere, contraddistinta da un «tono totalizzante» (2017: 12-13).

7. Agamben, cercando di ricostruire una genealogia del termine "dispositivo", prende le mosse da un'intervista di Foucault del 1977, nella quale l'autore esplicita la geometria complessa del concetto: «Ciò che io cerco di individuare con questo nome, è, innanzitutto, un insieme assolutamente eterogeneo che implica discorsi, istituzioni, strutture architettoniche, decisioni regolative, leggi, misure amministrative, enunciati scientifici, proposizioni filosofiche, morali e filantropiche, in breve: tanto del detto che del non-detto, ecco gli elementi del dispositivo. Il dispositivo è la rete che si stabilisce fra questi elementi...» (2006: 6).

8. In alcuni comuni (Tolentino, Pieve Torina, Muccia, Serravalle del Chienti) i sindaci hanno autorizzato l'installazione di strutture abitative temporanee - casette di legno o su ruote - onde evitare l'allontanamento della popolazione dalle proprie comunità. Tale comportamento è stato severamente sanzionato dalla Regione, attraverso l'ufficio per l'urbanistica, e dal Commissario Straordinario in quanto non conforme alle direttive emanate per la tutela dei terremotati e della fase di ricostruzione. Considerati i ritardi relativi all'assegnazione di SAE o container, questa forma di autogestione locale ha di fatto permesso in alcune località di evitare un'ulteriore dinamica di spopolamento.

9. Nick Dines (2016) riporta una dinamica analoga nel caso dell'Aquila, dove parte della popolazione si è riconosciuta - e ha cercato di rivendicare il suo status di cittadino aquilano - nel discorso sul patrimonio culturale e sul prestigio artistico della città. Egli spiega questo processo di "localizzazione" discorsiva dell'identità ricorrendo al concetto di "essenzialismo strategico" formulato dalla filosofa statunitense Gayatri Spivak -, un meccanismo che può essere attivato da un gruppo sociale in posizione di marginalità, il quale, essenzializzando alcune sue caratteristiche e appianando le differenze interne, rende più visibile e riconoscibile l'identità collettiva.

10. Jan-Jonathan Bock (2017) ha mostrato come, nel caso del terremoto aquilano del 2009, le politiche d'intervento dello Stato abbiano plasmato il senso collettivo degli eventi, tanto sul piano emotivo quanto su quello delle rappresentazioni inerenti al disastro. In particolare, egli nota come alla speranza indotta nei primi mesi dell'emergenza dal governo Berlusconi, attraverso la pronta reazione della Protezione Civile, sia seguita l'amara disillusione di una 
ricostruzione che, di fatto, non è mai davvero avvenuta per quanto riguarda il centro storico; effettivamente le soluzioni abitative adottate per l'Aquila, essendo state collocate in aree periferiche, hanno inesorabilmente ridisegnato in termini peggiorativi la socialità urbana, disgregando parzialmente la località e generando un sentimento di diffidenza nei confronti delle istituzioni radicalmente in contrasto con quello di fiducia mostrato inizialmente. La strategia dell'abbandono, che a ben vedere si concretizza a partire dall'assenza delle istituzioni, più che da una loro sparizione nel prolungarsi dell'emergenza, seguendo l'intuizione di Bock si configura in effetti come un campo del discorso in cui le ragioni esplicative sono dialetticamente legate all'esperienza emotiva del disastro, inteso come un processo in cui il ruolo dello Stato è carico di aspettative e responsabilità.

11. Si tratta della Rosfnet, azienda russa legata al progetto del gasdotto SNAM, la quale ha versato 5 milioni di euro per la ricostruzione dell'ospedale di Amandola.

\section{RIASSUNTI}

In questo articolo vengono ricostruite le rappresentazioni locali dei terremotati rispetto all'intervento delle istituzioni nel post-terremoto del Centro Italia. La "strategia dell'abbandono" rappresenta l'orizzonte comune delle interpretazioni fornite da alcune delle vittime in merito ai tragici eventi del 2016-2017. Attraverso il contributo dell'etnografia condotta sui luoghi del sisma, a circa un anno degli eventi, si cerca di spiegare la logica che guida l'azione delle istituzioni e l'attivazione del dispositivo d'emergenza - una logica caratterizzata dall'ambiguità. Le criticità emerse nel contesto del disastro sono infine ricondotte al concetto di "persuasione strutturale", la cui analisi qualitativa permette di esplicitare le interazioni locali e globali che, nel dispiegarsi della storia, sono in grado di plasmare, ridefinire e risignificare la memoria culturale degli eventi.

This essay analyzes the earthquake victim's representations of the emergency response of the State in the post-disaster phase after the Italian earthquake (2016-2017). The "neglect strategy" summarizes a common understanding of the events shared by some earthquake victims. On the basis of an ethnographic research led one year after the earthquake in some of the stricken areas, the article discusses both the ambiguous logic of the institutional strategy and the activation of the emergency dispositive. Critical issues observed in the context of the disaster are explained by the concept of "structural persuasion": a qualitative perspective enables the understanding of the local and global interactions that, as history unfolds, lead to important misrepresentations in the collective memory.

\section{INDICE}

Keywords : earthquake, Italy, structural persuasion, anthropology of disasters, emergency

Parole chiave : terremoto, Italia, persuasione strutturale, antropologia dei disastri, emergenza 


\section{AUTORE}

\section{FRANCESCO DANESI}

francescodanesi.amr@gmail.com 